\title{
The Utility and Validation of Intraepithelial Lymphocyte Count in Duodenal Biopsies in A Tertiary Care Centre in South India
}

\author{
Priyavadhana Balasubramanian', Bhawana Ashok Badhe ${ }^{1}$, Rajesh Nachiappa Ganesh*, \\ Lakshmi CPanicker $^{2}$ and Pazhanivel Mohan ${ }^{2}$ \\ 'Department of Pathology, JIPMER, Puducherry, India. \\ ${ }^{2}$ Department of Medical Gastroenterology, JIPMER, Puducherry, India.
}

\section{ABSTRACT}

Background: Intraepithelial lymphocytes (IELs) are seen in between the epithelial cells in small and large intestine with the functions of immune surveillance and activation.Increased IELs can be the sole histological finding in latent celiac disease (CD).

Aims: To study the utility and clinical relevance of IELs in diagnosing non-neoplastic lesions of duodenum and to determine a cut off for IEL to differentiate CD from other conditions.

Materials \& Methods: This was a prospective descriptive study.Duodenal biopsies from 101 patients with symptoms of malabsorption were studied. Informed written consent was taken. Clinical details were collected. Histomorphological parameters were studied on hematoxylin and eosin stained sections. Intraepithelial lymphocyte counts were done on CD3, CD4 and CD8 IHC stained sections. Statistical analysis was done using IBM- SPSS software version 21.P value $<0.05$ was considered statistically significant. The threshold score of IELs with maximum sensitivity and specificity was validated using the Receiver operator characteristic curve to distinguish CD from non-celiac disease conditions.

Results: We studied 101 duodenal biopsies. Our spectrum included 16 patients of CD (15.8\%), 15 of autoimmune duodenitis (14\%), 13 of nutritional deficiency associated duodenitis (12.8\%), 5 of infectious duodenitis (5\%) and 41 patients of non-specific duodenitis (40.6\%). The threshold levels were 5/20 villous tip IELs, 24 IELs/100 enterocytes on H\& E and >31 IELs by CD3 IHC staining.

Conclusion: Our study proposes IEL counts of $>31 / 100$ enterocytes in CD3 IHC staining to be significant in South Indian population to differentiate $\mathrm{CD}$ from other conditions.

\section{Keywords: Intraepithelial Lymphocytes, Duodenum, Celiac Disease, Immunohistochemistry.}

\section{Introduction}

Intraepithelial lymphocytes (IELs) are seen in between the epithelial cells in small and large intestine with the functions of immune surveillance and activation. Majority of them are T-cell type and express predominantly surface alpha/ beta $\mathrm{T}$ cell receptors. About $5 \%$ of the normal IELs express surface gamma/delta T-cell receptors which become the predominant population in celiac disease (CD). Increased IELs can be the sole histological finding in latent CD.[1]

The assessment of IEL counts have evolved since 1970s and the normal range for IEL count has been revised recently and it can be better assessed by immunohistochemistry with CD3 antibody. Presently, the normal IEL count in the proximal small intestine, especially in the duodenum is upto 25 IEL per 100 ECs.[2] Borderline intraepithelial lymphocytosis is considered when IEL count is between 26 to 29 on CD3 and definite intraepithelial lymphocytosis when it is $\geq 30 \mathrm{CD} 3$ positive IELs/100 ECs.[3,4]

Apart from celiac disease and tropical sprue, increased IELs in small bowel mucosa are seen associated with a variety of conditions like autoimmune disorders, tropical sprue, food protein intolerance, Helicobacter pyloriassociated gastritis, peptic duodenitis, parasitic and viral infections.[5 ]

We intended to study the utility and clinical relevance of intraepithelial lymphocytes in diagnosis of various nonneoplastic lesions of duodenum on endoscopic biopsies. And also aimed at determining if a cut off for IEL count can be determined to differentiate CD from other conditions.

\section{Materials \& Methods}

It is a descriptive study, conducted from May 2012 to June 2014 and 101 patients who presented with symptoms of malabsorption and evaluated with endoscopic duodenal biopsies were included in the study. The study was approved by the institutional review board and informed written consent was taken from the patients. Histopathological examination was carried out after standard processing \& staining procedures. Of the 101 cases, immunohistochemistry with CD 3 antibody was performed for 72 cases. CD4 and CD8 immunostaining 
was also done to study the ratio of $\mathrm{CD} 4$ and $\mathrm{CD} 8$ in various conditions.

Histomorphological parameters studied included villous architecture, crypt architecture, intraepithelial lymphocyte (IEL) count per 100 enterocytes (average of 300 enterocytes), IEL count using CD3, villous tip IEL count per 20 enterocytes were counted, inflammatory cells in lamina propria: type of cells - lymphocytes, neutrophils, eosinophils, epithelioid cells and its severity was graded as mild, moderate and severe. Modified Marsh Oberhuber classification was used to classify celiac disease.[6] Interpretation of IEL count and villous tip IEL count was done according to Datta Gupta et al. [7] Statistical analysis was done using IBM- SPSS software version 21. All statistical tests were carried out at $5 \%$ level of significance and $\mathrm{P}$ value $<0.05$ was considered statistically significant. An appropriate cut off for IEL counts in H \& E stained sections and on CD3 sections and villous tip IEL counts for celiac disease and non-celiac disease conditions was deduced using Receiver Operator Characteristic (ROC) curve in our study.

\section{Results}

We studied 101 duodenal biopsies during a 2 year study period. The various categories of patients included 16 cases $(15.8 \%)$ of celiac disease, 15 cases $(14.9 \%)$ of duodenitis associated with autoimmune diseases, 13 cases (12.8\%) of nutritional deficiency associated duodenitis, five cases $(5 \%)$ of infectious duodenitis, 41 cases $(40.6 \%)$ of nonspecific duodenitis and 11 cases $(10.9 \%)$ of miscellaneous duodenitis.

Villous architecture was studied as normal, mild, moderate or severe blunting. All cases of CD had villous blunting of varying degrees and were associated with crypt hyperplasia and either total or partial villous atrophy. Only nine out of 85 patients in non-celiac disease conditions had mild villous blunting.

IEL count was expressed per 100 enterocytes taking an average of 300 enterocytes. All patients of CD had increased IEL counts in the range of 25-45 IEL per 100 enterocytes and mean IEL counts of $28.2 \pm 7.7$. Villous tip IELs was calculated per 20 villous tip enterocytes by averaging the counts for five villi. Villous tip IEL was categorised as normal and increased based on already established cut off of 5 IELs per 20 enterocytes. The range of villous tip IEL counts in CD was 3-14 IELs per 20 enterocytes whereas in other disease groups it ranged from 0-12 IELs per 20 enterocytes. Mean villous tip IEL counts for $\mathrm{CD}$ was $8.62 \pm 3.14$ which was higher compared to nonceliac disease conditions. [Fig 1(a), (b), (c)].
Comparison of IEL count between H\&E and CD3 IHC staining:

Normal IEL counts was found in $65 / 72$ cases on H\&E stained sections. Of these 65 cases, when CD3 IHC staining was performed, 40/65 cases had normal IEL counts, 9 cases had borderline increased and 16 cases had increased IEL counts. Borderline increased IEL counts was seen in 2/72 cases on H\&E but had increased IELs on CD3. However $5 / 72$ patients had similar counts of definitely increased IELs in both $\mathrm{H} \& \mathrm{E}$ and $\mathrm{CD} 3 \mathrm{IHC}$ stained sections.

The level of agreement was done using kappa statistics between IEL counts on H\& E stained sections and CD 3 stained sections. Kappa value was 0.207 concluding that there is no correlation and it was statistically significant with $p$ value less than 0.001 . Hence quantification of IEL by $\mathrm{CD} 3$ has much better outcome. In our study, 25 patients (24.75\%) of normal counts by H\& E, shifted to either borderline or definitely increased. [Fig 1 (d)].

Mean of CD8: CD4 was was similar in all groups, indicating that CD8 suppressor cells predominated over helper T cells in all disease conditions.

Receiver operator characteristic (ROC) curve for validation of IEL counts in duodenal biopsies:

IEL counts on $\mathrm{H} \& \mathrm{E}$ stained sections: At a threshold of 24 IELs/100 enterocytes, the sensitivity was $81.2 \%$ and specificity $80 \%$, which was similar to the already established cut off. Though the sensitivity of $81.2 \%$ was seen from a range of 22.5 to 24 IELs per 100 enterocytes but maximum specifity was seen at 24 IELs per 100 enterocytes for our study population. Area under the curve was 0.865 which was significant.

IEL counts on CD 3 IHC stained sections: Maximum sensitivity and specificity was seen at a cut off of 31 IELs/100 enterocytes with sensitivity of $83.3 \%$ and specificity of $79.8 \%$ in distinction of $\mathrm{CD}$ from other diseases. The sensitivity of $83.3 \%$ was seen from a cut of $25.5 \% \mathrm{IELs} / 100$ enterocytes, but the specificity was very low starting from $62.1 \%$. The area under the curve was 0.872 which was statistically significant. When we applied the cut off of 31 IELs per 100 enterocytes in our cases, only 3 cases of non specific duodenitis had increased IEL counts compared to 10 cases when using the earlier cut off.

Villous tip IEL counts: Maximum sensitivity of $87.5 \%$ and maximum specificity of $71.8 \%$ was found at a cut off of 5 IELs per 20 enterocytes and maintained till 5.5 IELs per 20 enterocytes. The area under the curve was 0.837 which was statistically significant. 
Table 1 shows the sensitivity and specificity at various cut off of IELs on H \& E, CD 3 stained sections and villous tip IELs.
Table 2 shows the representation of IELs by CD3 in various groups after validation by ROC curve.

Table 1: Sensitivity and specificity at various cut off of IELs on H \& E, CD 3 stained sections and villous tip IELs.

\begin{tabular}{|c|c|c|c|c|c|c|c|c|}
\hline \multicolumn{2}{|c|}{ H \& E stained sections. } & \multicolumn{2}{c|}{ CD 3 stained sections. } & \multicolumn{4}{c|}{ Villous tip IELs } \\
\hline $\begin{array}{c}\text { Cut off of } \\
\text { IELs }\end{array}$ & Sensitivity & Specificity & $\begin{array}{c}\text { Cut off of } \\
\text { IELs }\end{array}$ & Sensitivity & Specificity & $\begin{array}{c}\text { Cut off of villous } \\
\text { tip IELs }\end{array}$ & Sensitivity & Specificity \\
\hline 19.5 & 87.5 & 72.9 & 25.50 & 83.3 & 62.1 & 3.50 & 93.8 & 52.9 \\
\hline 21.00 & 81.2 & 75.3 & 26.50 & 83.3 & 68.2 & 4.50 & 87.5 & 64.7 \\
\hline 22.50 & 81.2 & 78.8 & 27.50 & 83.3 & 69.7 & $\mathbf{5}$ & $\mathbf{8 7 . 5}$ & $\mathbf{7 1 . 8}$ \\
\hline $\mathbf{2 4 . 0 0}$ & $\mathbf{8 1 . 2}$ & $\mathbf{8 0}$ & 29.00 & 83.3 & 72.7 & $\mathbf{5 . 5 0}$ & $\mathbf{8 7 . 5}$ & $\mathbf{7 1 . 8}$ \\
\hline 25.50 & 68.8 & 83.5 & $\mathbf{3 1 . 0 0}$ & $\mathbf{8 3 . 3}$ & $\mathbf{7 8 . 8}$ & 6.50 & 75.0 & 74.1 \\
\hline 26.50 & 68.8 & 84.7 & 32.50 & 66.7 & 81.8 & 7.50 & 62.5 & 82.4 \\
\hline
\end{tabular}

IELs- Intraepithelial lymphocytes $H \& E$ - haemotoxylin and eosin

Table2 : Representation of IELs by CD3 in various groups after validation by receiver operator characteristic curve.

\begin{tabular}{|l|c|c|c|c|c|c|}
\hline \multirow{2}{*}{ Disease conditions } & \multicolumn{2}{|c|}{$\begin{array}{c}\text { Before \& after validation } \\
\text { IEL counts by H\&E }\end{array}$} & $\begin{array}{c}\text { After validation IEL counts } \\
\text { by CD3 }\end{array}$ & \multicolumn{2}{|c|}{$\begin{array}{c}\text { Before validation IEL } \\
\text { counts by CD3 }\end{array}$} \\
\cline { 2 - 7 } & $\begin{array}{c}\text { Normal } \\
(\leq 24)(n *, \%)\end{array}$ & $\begin{array}{c}\text { Increased } \\
(>24)(n, \%)\end{array}$ & $\begin{array}{c}\text { Normal } \\
(\leq 31)(n, \%)\end{array}$ & $\begin{array}{c}\text { Increased } \\
(>31)(n, \%)\end{array}$ & $\begin{array}{c}\text { Normal } \\
(\leq 24(n, \%)\end{array}$ & $\begin{array}{c}\text { Increased } \\
(>25)(n, \%)\end{array}$ \\
\hline Autoimmune duodenitis (14) & $12(85.7)$ & $2(14.3)$ & $9(64.3)$ & $5(35.7)$ & $8(57.1)$ & $6(42.8)$ \\
\hline Celiac disease (6) & $2(33.3)$ & $4(66.7)$ & $1(16.7)$ & $5(83.3)$ & 0 & $6(100)$ \\
\hline $\begin{array}{l}\text { Nutritional deficiency } \\
\text { associated duodenitis (11) }\end{array}$ & $10(90.9)$ & $1(9.1)$ & $9(81.8)$ & $2(18.2)$ & $6(54.5)$ & $5(45.4)$ \\
\hline Non specificduodenitis (31) & $31(100)$ & 0 & $28(90.3)$ & $3(9.7)$ & $21(67.7)$ & $10(32.2)$ \\
\hline Infectious duodenitis (3) & $2(66.7)$ & $1(33.3)$ & $2(66.7)$ & $1(33.3)$ & $2(66.7)$ & $1(33.3)$ \\
\hline
\end{tabular}

$*_{n}$ - number of cases

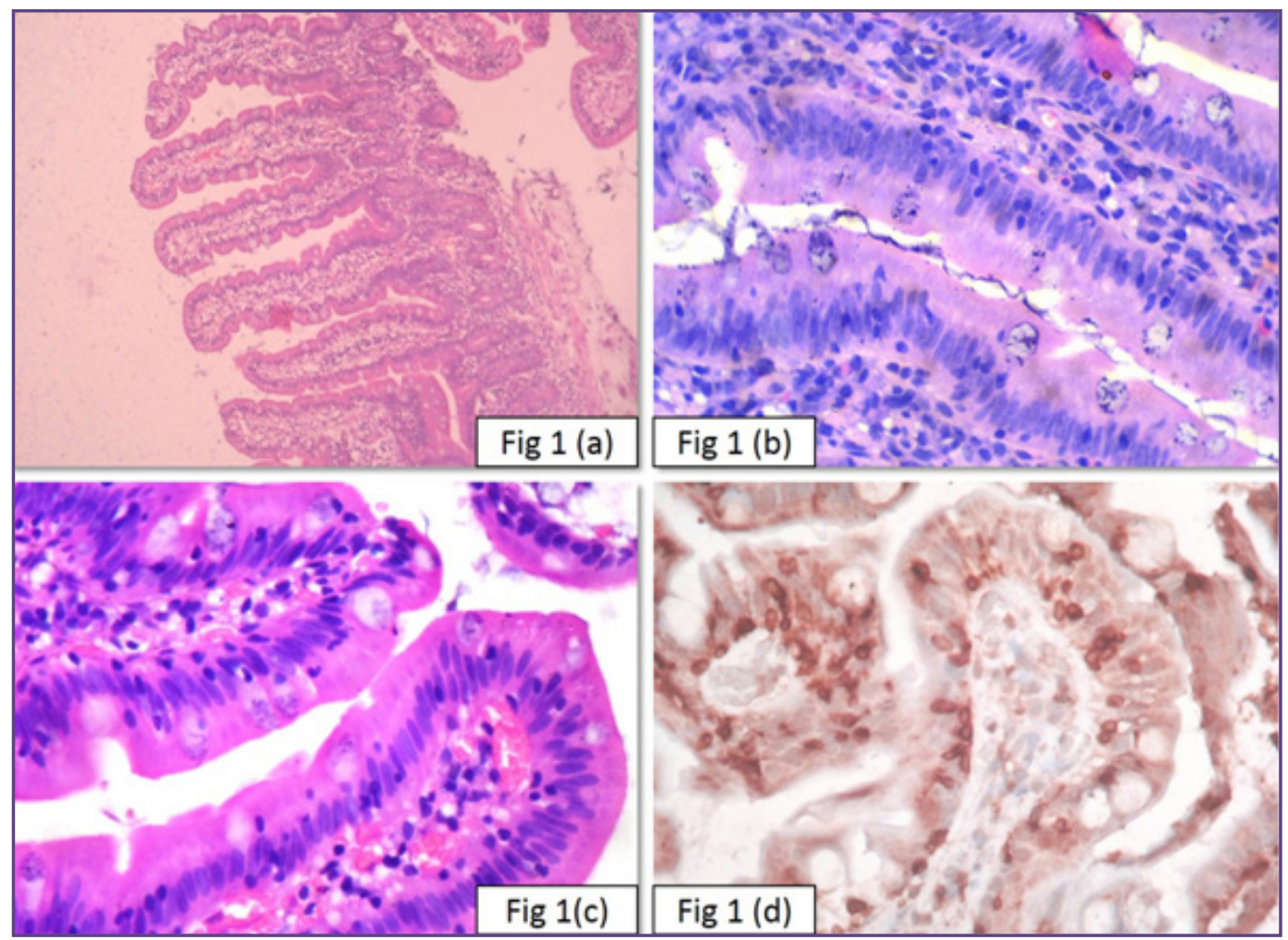

Fig. 1 (a): Well oriented villi with villous crypt ratio of 3-5:1(H\&E,40 X).Fig 1 (b): Villi showing increased IELs (H\&E, 400 X).Fig 1 (c): Villous tip showing increased IELS (H\&E, 400 X).Fig 1 (d): Villi showing increased IELs (CD 3,DAKO, 400 X). 


\section{Discussion}

This study was undertaken to know the spectrum of malabsorptive disorders of small intestine in South Indian population and to study the significance of IEL and validate the IEL count with maximum sensitivity and specificity in to distinguish of CD from non-celiac disease conditions.

Celiac disease is one of the common causes of malabsorption in various wheat consuming parts of the world including North India. Very few studies especially case reports and case series are available from South India on CD. All the studies done across different parts of the world reported the prevalence of CD ranging from 0.14 to $1.17 \%$.[8-21] In our study, CD constituted $15.8 \%$ of patients with malabsorption who underwent small intestinal biopsies.

The other cases reported in our study were 15 patients (14\%) with autoimmune duodenitis. nutritional deficiency associated duodenitis was seen in 13 patients (12.8\%), infectious duodenitis was seen in 5 patients $(5 \%)$ while non specific duodenitis where no etiological agent was found with clinicopathologic correlation was seen in 41 patients, constituting $40.6 \%$ of patients. [22]

Histological parameters of villous blunting, atrophy, crypt hyperplasia, increased IEL counts are characteristic in CD but not specific. In our study, villous crypt hyperplasia, villous blunting, villous atrophy, villous tip IEL $(>5 / 20$ enterocytes) and increased IELs ( $>25 / 100$ enterocytes) were statistically significant in distinction of CD from nonceliac disease conditions by univariate analysis. However, we could not evaluate these parameters for statistical significance by multivariate analysis in view of the smaller numbers in each group.

Increased IEL counts is one of the important histological feature seen in $\mathrm{CD}$ along with villous atrophy. But increased IEL counts in architecturally normal duodenal biopsies ranged from $9 \%$ to $40 \%$ in various studies. $[1,25,26]$ It is important to report it as these patients may be in latent phase of CD and are likely to develop CD in future necessitating close monitoring.

In our study, we had 37 patients $(36.6 \%)$ of non-celiac disease with increased IELs. Other causes of increased IEL counts in our study were 6 patients $(46.7 \%)$ of autoimmune duodenitis, 6 patients $(46.2 \%)$ of nutritional deficiency associated duodenitis, $60 \%$ of infectious duodenitis and $39 \%$ of non-specific duodenitis. The distribution in our study was comparable with the other studies.[1,27]

Biagi et al counted IEL in villous tips and mentioned in their study that villous tip IEL is sufficient for diagnosing CD. [28] In this study, the mean villous tip IEL scores were
4.6 with range of 1.4-7.8 in non-celiac diseases compared to 9.2 with range of $5.8-21.8$ in patients with $\mathrm{CD}$. They concluded that counting villous tip IELs is very simple and quite reliable method. Jarvinen TT et al in their study mentioned that the villous tip IEL counts was significantly higher in early celiacs.[29] Further in their study, the sensitivity of villous tip IELs was 0.84 and the specificity was 0.88 in detecting untreated celiacs especially with no villous abnormalities. Also, they reported villous tip IELs to be superior to CD3+ IEL counts. Also they mentioned that villous tip IELs seems to be similar to gamma/delta+ cells which can be done only on frozen sections.

In our study, the range of villous tip IELs was from 3 to 14 per 20 enterocytes with mean count of 9.2 IELs per 20 enterocytes in celiac patients compared to non celiac disease conditions where it ranges from 0 to 12 with mean count of 4.2 IELs per 20 enterocytes and this was statistically significant. Our findings were similar to the study done by Biagi et al.[28 ]We can conclude that increased villous tip IELs can help to distinguish CD from other close mimics, thus provides a valuable tool in routine practice.

A cut off threshold of 5 per 20 enterocytes is suggested in the published literature to identify $C D$. In view of the low prevalence in our study population, we studied the strength of threshold levels of villous tip IELs in distinguishing $\mathrm{CD}$ from non-celiac disease conditions, based on Receiver Operating Characteristic (ROC) curve. We found that a threshold of 5/20 villous tip IELs had a sensitivity of 87.5 with specificity of 71.76 and this level was maintained up to $5.5 / 20$ villous tip IELs. Beyond this threshold, the sensitivity for diagnosing $\mathrm{CD}$ decreases while the specificity increases. Thus, the threshold of 5 villous tip IEL/20 enterocytes holds good for low prevalence setting of $\mathrm{CD}$ in South India.

CD3 immunohistochemistry aids the detection of IELs much easier as some IELs have irregular nuclear outline, and some may resemble epithelial cells. In the study done by S.Nasseri-Moghaddam et al, the IEL count was slightly higher in IHC than H\&E (21 vs 19) and the two methods showed excellent agreement statistically.[30] In the study done by Pellegreno et al, there was high correlation of IEL counts between H\& E and CD3 staining which was statistically significant. [23] In our study, there was no correlation with H\& E and CD3 staining for IEL counts and it was statistically significant. Sixteen patients with normal IEL on $\mathrm{H} \& \mathrm{E}$ staining had increased counts when CD3 immunostaining was applied. This study further highlights the importance of CD 3 staining which can pick up near normal patients.

In our study, we validated the threshold of IELs in 
distinguishing $\mathrm{CD}$ from non-celiac disease conditions by charting the values in ROC curve. We found that at a threshold of 24 IELs/100 enterocytes, the sensitivity is $81.2 \%$ and specificity of $80 \%$ were at their peak thus confirming to the existing literature. Though the sensitivity of $81.2 \%$ was seen from 22.5 to $24 / 100$ enterocytes, but maximum specificity was seen at cut of 24 per 100 enterocytes. However, for CD3 immunohistochemistry the threshold was higher at 31 IELs/100 enterocytes with sensitivity of $83.3 \%$ and specificity of $79.8 \%$ in the distinction of $\mathrm{CD}$ from other diseases. For $\mathrm{CD} 3$, the sensitivity of $83.3 \%$ was seen from a cut of $25.5 \%$ IELs/ 100 enterocytes but the specificity was very low starting from $62.1 \%$.

Thus in our study, we recommend CD3 immunostaining, as it is statistically significant in comparison with $\mathrm{H} \& \mathrm{E}$ staining with a kappa score of 0.207 in identifying IELs. Hence by using a higher threshold of IELs by CD3 immunostaining, sensitivity increased.

In our series we studied expression of CD4 and CD8 in intraepithelial lymphocytes in 73 patients. The average CD8:CD4 ratio was 6.64 ranging from 6.4 to 6.7. In all the conditions, predominant cell population in the intraepithelial lymphocytes were $\mathrm{T}$ suppressor group and there was no difference in $\mathrm{CD} 8: \mathrm{CD} 4$ ratio in $\mathrm{CD}$ and nonceliac disease group.

\section{Conclusion}

We have validated the increase in IEL count using $\mathrm{H} \& \mathrm{E}$ staining and $\mathrm{CD} 3$ immunostaining. Our findings strengthen the cut off threshold of $>25 / 100$ enterocytes for increased IEL and $>5 / 20$ enterocytes in villous tip IEL using H\&E staining. With CD3 immunostaining, the threshold shift of 31/100 enterocytes as increased IELs was found to increase the specificity in diagnosing $\mathrm{CD}$.

\section{Acknowledgment}

I would like to acknowledge, Dr. Archana R, Senior Resident,Department of Preventive \& social medicine, JIPMER for helping in statistical analysis of this study.

\section{References}

1. Mahadeva S, Wyatt JI, Howdle PD. Is a raised intraepithelial lymphocyte count with normal duodenal villous architecture clinically relevant? J Clin Pathol. 2002;55:424-8.

2. Hayat M, Cairns A, Dixon MF, O’Mahony S. Quantitation of intraepithelial lymphocytes in human duodenum: what is normal? J Clin Pathol. 2002;55:393-4.

3. Veress B, Franzén L, Bodin L, Borch K. Duodenal intraepithelial lymphocyte-count revisited. Scand J Gastroenterol. 2004;39:138-44.

4. Cabanne A, Vázquez H, Argonz J, et al. Clinical utility of counting intraepithelial lymphocytes in celiac disease intestinal mucosa. Acta Gastroenterol Latinoam. 2007;37:20-8.

5. Chang F, Mahadeva U, Deere H. Pathological and clinical significance of increased intraepithelial lymphocytes IELs in small bowel mucosa. APMIS. 2005;113:385-9

6. Kalhan S, Joseph P, Sharma S, Dubey S, Dudani S, Dixit M. Comparative study of histopathological Marsh grading with clinical and serological parameters in celiac iceberg of north India. Indian J Pathol Microbiol.2011;54:279-83.

7. GS Datta. Pathology of celiac disease: a brief review. Trop Gastroenterol 2013;34:207-26.

8. Sher KS, Frasar RC, Wicks AC, Mayberry JF. High risk of celiac disease in Punjabis : Epidemiological study in the south Asian and European populations of Leicestershire. Digestion. 1993;54:178-82.

9. Sood A, Midha V, Sood N, Avasthi G, Sehgal A. Prevalence of celiac disease among school children in Punjab, North India. J Gastroenterol Hepatol.2006;21:1622-5.

10. Bhattacharya M, Dubey AP, Mathur NB. Prevalence of celiac disease in north Indian children. Indian Pediatr. 2009;46:415-7.

11. Tatar G, Elsurer R, Simsek H, et al. Screening of tissue transglutaminase antibody in healthy blood donors for celiac disease screening in the Turkish population. Dig Dis Sci. 2004;49:1479-84.

12. Ertekin V, Selimoğlu MA, Kardaş F, Aktaş E. Prevalence of celiac disease in Turkish children. J Clin Gastroenterol.2005;39:689-91.

13. Akbari MR, Mohammadkhani A, Fakheri H, et al. Screening of the adult population in Iran for coeliac disease: comparison of the tissue-transglutaminase antibody and anti-endomysial antibody tests. Eur J Gastroenterol Hepatol. 2006;18:1181-6.

14. Sood A, Midha V, Sood N, Malhotra V. Adult celiac disease in northern India. Indian J Gastroenterol. 2003;22:124-6.

15. Abu-Zekry M, Kryszak D, Diab M, Catassi C, Fasano A. Prevalence of celiac disease in Egyptian children disputes the east-west agriculture-dependent spread of the disease. J Pediatr Gastroenterol Nutr.2008;47:136-40.

16. Sachdev A, Srinivasan V, Maheswary S, Mohan H, Ashish B, Singh LS. Adult onset celiac disease in north India. Trop Gastroenterol. 2002;23:117-9.

17. Ben Hariz M, Kallel-Sellami M, Kallel L, et al. Prevalence of celiac disease in Tunisia: mass-screening study in school children. Eur J Gastroenterol Hepatol. 2007;19:687-94.

18. Ramakrishna BS. Celiac disease: can we avert the impending epidemic in India? Indian J Med Res. 2011;133:5-8

19. Yachha SK, Poddar U. Celiac disease in India. Indian J Gastroenterol. 2007;26:230-7.

20. Ganesh R, Suresh N, Sathiyasekaran M. Celiac disease, still an uncommon problem in Tamilians? Indian J Gastroenterol. 2009;28:189.

21. Shamir R, Lerner A, Shinar E,et al. The use of a single 
serological marker underestimates the prevalence of celiac disease in Israel: a study of blood donors. Am J Gastroenterol.2002;97:2589-94.

22. Balasubramanian P, Badhe BA, Ganesh RN, Panicker LC, Mohan P. Morphologic Spectrum of Duodenal Biopsies in Malabsorption: A Study from Southern India. J Clin Diagn Res. 2017;11:17-21.

23. Pellegrino S, Villanacci V, Sansotta N, et al. Redefining the intraepithelial lymphocytes threshold to diagnose gluten sensitivity in patients with architecturally normal duodenal histology. Aliment Pharmacol Ther.2011;33: 697-706.

24. Brown I, Mino-Kenudson M, Deshpande V, Lauwers GY. Intraepithelial Lymphocytosis in Architecturally Preserved Proximal Small Intestinal Mucosa. An Increasing Diagnostic Problem With a Wide Differential Diagnosis. Arch Pathol Lab Med. 2006;130:1020-5.

25. Kakar S, Nehra V, Murray JA, Dayharsh GA, Burgart LJ. Significance of intraepithelial lymphocytosis in small bowel biopsy samples with normal mucosal architecture. Am J Gastroenterol. 2003;98:2027-33.

26. Wahab PJ, Crusius JB, Meijer JW, Mulder CJ. Gluten challenge in borderline gluten sensitive enteropathy. Am J Gastroenterol. 2001;96:1464-9.

27. Williams L, Dew MJ, Murray LA, Williams DA. Are routine duodenal biopsies taken at the time of an upper GI endoscopy clinically useful? Gasteroenterol Today. 2001;11:73-76.

28. Biagi F, Luinetti O, Campanella $\mathrm{J}$, et al. Intraepithelial lymphocytes in the villous tip: do they indicate potential coeliac disease? J Clin Pathol. 2004;57:835-9.

29. Järvinen TT, Collin P, Rasmussen $M$, et al. Villous tip intraepithelial lymphocytes as markers of early-stage coeliac disease. Scand J Gastroenterol. 2004; 39:428-33.

30. Nasseri-Moghaddam S, Mofid A, Nouraie M, et al. The normal range of duodenal intraepithelial lymphocytes. Arch Iran Med. 2008;11:136-42.

*Corresponding author:

Dr. Rajesh Nachiappa Ganesh, Additional Professor, Department of Pathology, JIPMER, Puducherry-605006, India.

Phone: +91 09791976021

Email: drngrajesh@gmail.com.

Financial or other Competing Interests: None. 